\title{
REVISIONES
}

\section{Terapia nutricional y pre-habilitación multimodal en el paciente quirúrgico no crítico. Una revisión.}

Nutritional therapy and multimodal pre-habilitation in the non-critical surgical patient. A review.

\author{
Eduardo Moreira ${ }^{1}$, Patricia López ${ }^{2}$, Lourdes Silva ${ }^{3}$, Estela Olano ${ }^{4}$
}

DOI: $10.31837 /$ cir.urug/2.2.5

Recibido: 14 de febrero de 2018

Aceptado: 23 de agosto de 2018

\section{Resumen}

Los pacientes quirúrgicos a menudo tienen un alto riesgo nutricional y esto se asocia con resultados posoperatorios deficientes, poniendo la supervivencia en riesgo.La identificación precoz de este tipo de pacientes es esencial para las intervenciones nutricionales tempranas con el objetivo de atenuar el catabolismo y preservar los procesos de respuesta al estrés quirúrgico que promueven la recuperación e inmunoprotección, reducen la morbimortalidad perioperatoria y mejoran los resultados quirúrgicos.En los últimos años, se han estudiado diversas estrategias nutricionales durante el período perioperatorio que han mostrado beneficios.El objetivo de la presente revisión es analizar la evidencia más reciente sobre la terapia nutricional en pacientes quirúrgicos no críticos; para ello se han consultado bases de datos electrónicas como PubMed, y revistas de las principales sociedades científicas competentes en la materia.

Palabras clave: Terapia nutricional, cirugía, riesgo nutricional, inmunonutrición, ayuno preoperatorio, prehabilitación en cirugía.

\begin{abstract}
Surgical patients often have a high nutritional risk and this is associated with poor postoperative results, putting survival at risk.The early identification of this type of patients is essential for early nutritional interventions with the aim of attenuating catabolism and preserving the processes of response to surgical stress that promotes recovery and immunoprotection, reduces perioperative morbidity and mortality and improves surgical outcomes.In recent years, various nutritional strategies have been studied during the perioperative period that have shown benefits. The purpose of the present review is to analyze the most recent evidence on nutritional therapy in non-critical surgical patients; for this purpose, electronic databases such as PubMed and journals of the main scientific societies competent in this field have been consulted.
\end{abstract}

Key words: Nutrition therapy, Surgery.Nutritional risk, Immunonutrition, Preoperative fasting, Prehabilitation in surgery.

\footnotetext{
${ }^{1}$ Médico Intensivista. UCI del Hospital Maciel. Posgrado en Soporte Nutricional Avanzado. Integrante del Equipo Multidisciplinario de Terapia Nutricional del Hospital Maciel. ASSE. Montevideo, Uruguay. dreduardo.moreira@gmail.com

${ }^{2}$ Prof. Adj. de Clínica Quirúrgica. Hospital de Clínicas. Facultad de Medicina. UdelaR. Integrante del Equipo Multidisciplinario de Terapia Nutricional del Hospital Maciel. ASSE. Montevideo, Uruguay.

${ }^{3}$ Licenciada en Enfermería Especializada.. Integrante del Equipo Multidisciplinario de Terapia Nutricional del Hospital Maciel. ASSE. Montevideo, Uruguay.

${ }^{4}$ Médico Intensivista. Coordinadora del Equipo Multidisciplinario de Terapia Nutricional del Hospital Maciel. ASSE. Montevideo, Uruguay.
} 
Se realizó una revisión exhaustiva de tipo cualitativo y crítico de la literatura médica actualizada, con búsqueda bibliográfica en revistas de las principales sociedades científicas relacionadas y en base de datos PubMed y Medline en los idiomas inglés, español y francés. Engloba los principales tópicos de la terapia nutricional en el paciente quirúrgico y ofrece al cirujano tratante un resumen del conocimiento científico actualizado que facilitará su desempeño y contribuirá con la optimización de los resultados en cirugía.

\section{Introducción}

\section{Importancia de la desnutrición en el paciente quirúrgico.}

La prevalencia de desnutrición en cirugía es variable ${ }^{12}$, según el estudio multi-céntrico español de Planas y otros. ${ }^{3}$ La desnutrición en el paciente quirúrgico hospitalizado es del 10\%, sin embargo, otros autores han encontrado que el 65\% de los pacientes que se realizan una cirugía del tracto gastrointestinal (TGI) superior presentan algún grado de desnutrición y esta condición puede afectar hasta al 80\% de los pacientes con cáncer de cabeza y cuello o cáncer de páncreas ${ }^{4}$. El estudio IBRANUTRI que evaluó a 4000 pacientes de Brasil evidenció que la desnutrición está presente en el 48,1\%, siendo 12,6\% desnutridos graves y 35,5\% desnutridos moderados ${ }^{5}$. En una revisión sistemática reciente que incluyó a 66 estudios latinoamericanos, Correia y cols. constataron alta prevalencia de desnutrición, sobre todo en pacientes añosos, críticos y quirúrgicos ${ }^{6}$.

La pérdida de peso durante una estancia hospitalaria mayor a 7 días, independientemente del estado nutricional al ingreso, se asocia con un aumento de los costos y las complicaciones ${ }^{7}$. Esta pérdida de peso adicional se produce en más del $60 \%$ de los pacientes, y es secundaria a múltiples factores, como se observan en la tabla 1.

Tabla 1: Causas de desnutrición hospitalaria en el paciente quirúrgico ${ }^{89}$.

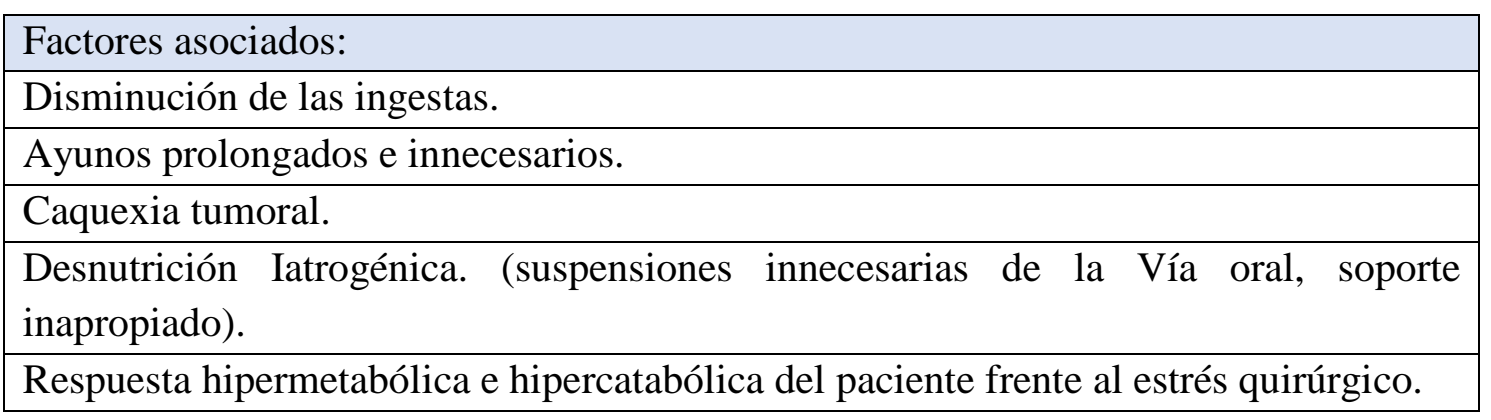


Una revisión sistemática de 15 estudios en pacientes ancianos evidenció que la pérdida de peso y la concentración de albúmina sérica eran parámetros predictivos para el resultado posoperatorio ${ }^{10}$, datos que se confirmaron en el estudio de Aahlin, y otros en $2015^{11}$. La mayoría de los pacientes en riesgo nutricional se encuentran en los servicios de cirugía, oncología, geriatría y cuidados intensivos. Sorensen, y otros. demostraron que el nivel de gravedad de la enfermedad, una edad superior a 70 años, cirugía y cáncer impactan significativamente en la tasa de complicaciones hospitalarias ${ }^{12}$. Las tres razones más comunes para el reingreso hospitalario 30 días después de una cirugía general son complicaciones gastrointestinales, infecciones quirúrgicas y desnutrición ${ }^{13}$.

El trauma quirúrgico induce un estado de estrés que amenaza la homeostasis si esa respuesta se perpetúa. Las terapias clínicas deberían por lo tanto apuntar a atenuar el catabolismo mientras se preservan los procesos de respuesta al estrés quirúrgico que promueven la recuperación y la inmunoprotección ${ }^{14,15}$.

La terapia nutricional y la pre-habilitación adecuada (integrada al manejo general del paciente) durante el perioperatorio pueden reducir las complicaciones, acortar la hospitalización y disminuir la necesidad de cuidados intensivos. En consecuencia, puede ser una intervención altamente costoeficiente $^{16,17,18}$

\section{Terapia Nutricional en el paciente quirúrgico no crítico, hospitalizado.}

\section{Antes de la cirugía. Pre-habilitación nutricional.}

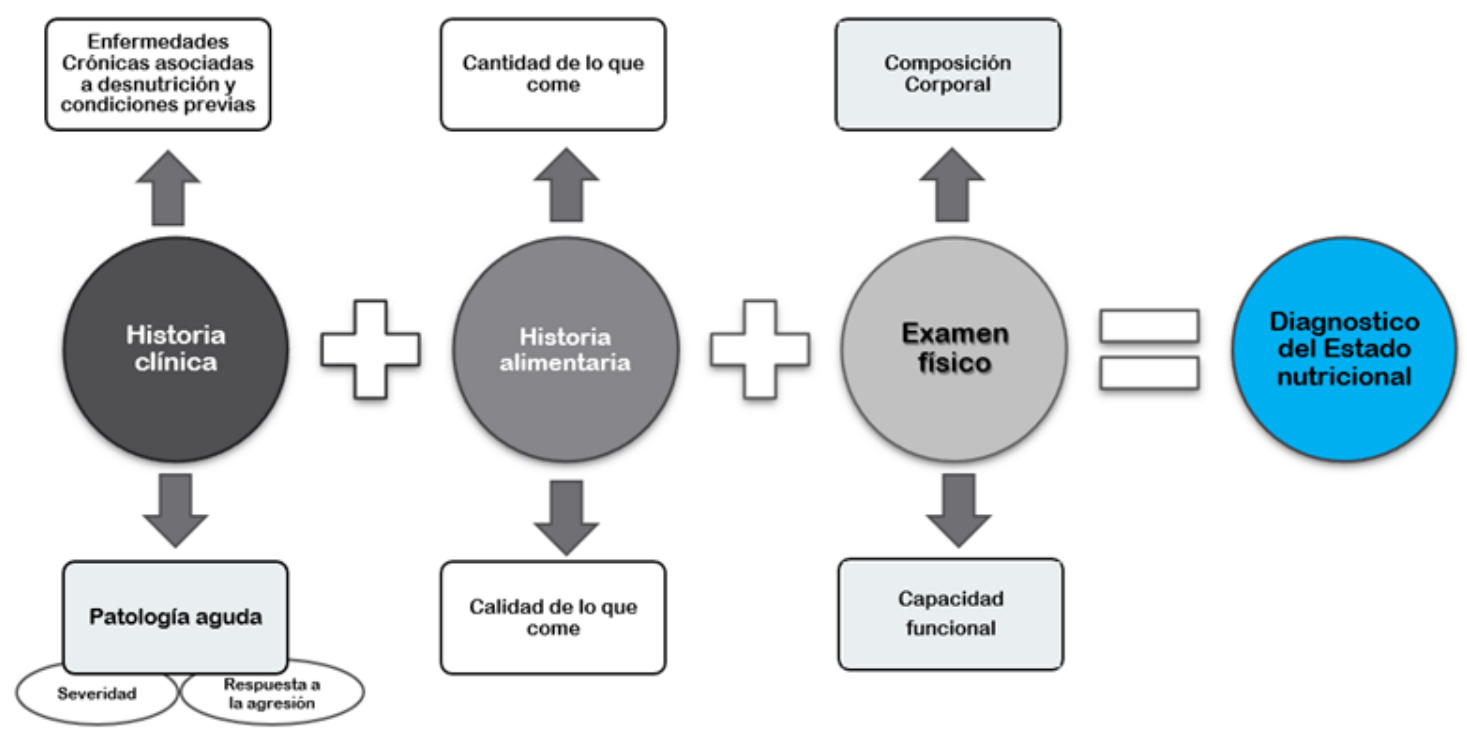

Fig 1. Diagnóstico del estado nutricional. 
La valoración nutricional previo a la cirugía es esencial para el manejo del paciente quirúrgico. Algunos de los instrumentos más conocidos, validados por ESPEN (EuropeanSocietyfor Parenteral and Enteral Nutrition) e indicados para valoración nutricional son presentados en la tabla 2.

Tabla 2: Herramientas para valoración Nutricional en el paciente quirúrgico ${ }^{19}$.

\begin{tabular}{|l|}
\hline Instrumentos. \\
\hline SGA (Subjective Global Assessment) \\
\hline MUST (Malnutrition Universal Screening Tool) \\
\hline NRI (Índice de Riesgo Nutricional) \\
\hline MNA (Mini-NutritionalAssessment) \\
\hline MST (Malnutrition Screening Tool) \\
\hline NRS 2002 (NutritionRisk Screening) \\
\hline NUTRIC Score (Nutrition Risk in Critically Ill) \\
\hline
\end{tabular}

Las indicaciones para la terapia nutricional son la prevención y el tratamiento del catabolismo, la malnutrición y el mantenimiento perioperatorio del estado nutricional para prevenir las complicaciones postoperatorias de aquellos pacientes con riesgo nutricional. Por lo tanto, se debe considerar el cribado sistemático del riesgo nutricional en todos los pacientes al ingreso hospitalario ${ }^{20}$.

Las concentraciones bajas de albúmina sérica preoperatoria están asociadas con retrasos en cicatrización de la herida y pueden predecir la morbilidad en pacientes sometidos a cirugías electivas ${ }^{2122}$. No obstante, como la concentración de albúmina es suprimida por la cirugía y la enfermedad, su medición perioperatoria tiene un valor limitado.El valor clínico de este y otros marcadores es sugestivo más que diagnóstico, ya que no son específicos para malnutrición ${ }^{23}$.

La clave en el cuidado nutricional perioperatorio es definir el riesgo nutricional, que es cercano al 47\% en pacientes de cirugía abdominal, en la tabla 3 se presentan algunos criterios para definirlo, el NRS 2002 es la herramienta mejor validada para este propósito en pacientes quirúrgicos ${ }^{24}$, en una revisión sistemática de la literatura que incluyó diez estudios, Gupta y otros. ${ }^{25}$ pusieron en evidencia que el estado nutricional del paciente medido con una herramienta nutricional validada es un buen predictor de la duración de la estancia hospitalaria en pacientes con cáncer gastrointestinal que requieren cirugía, sugieren que el soporte nutricional puede disminuir la estancia y las readmisiones hospitalarias.

Tabla 3: Criterios para determinar Riesgo nutricional elevado.

- $\quad$ Pérdida de peso $>10$ a15\% dentro de los 6 meses

- $\mathrm{IMC}<18.5 \mathrm{~kg} / \mathrm{m}^{2}$

- Valoración Global Subjetiva Grado C o NRS $2002>5$

- Albúmina sérica preoperatoria <3.0 g / l (sin evidencia de disfunción hepática o renal).

(Con la presencia de al menos uno de los criterios se establece riesgo nutricional elevado) 
ESPEN ha definido recientemente criterios de diagnóstico para la malnutrición de acuerdo con dos opciones que se esquematizan en la Tabla $4^{26}$.

Tabla 4: Definición de Desnutrición Relacionada con la Enfermedad

\begin{tabular}{|l|l|l|l|}
\hline $\begin{array}{c}\text {. Definición } \\
\text { de DRE de } \\
\text { ESPEN }\end{array}$ & $\begin{array}{l}\text { Reducción del } \\
\text { Índice de Masa } \\
\text { Corporal } \\
\text { (IMC) } *\end{array}$ & $\begin{array}{l}\% \text { de Pérdida de } \\
\text { peso corporal }\end{array}$ & $\begin{array}{l}\text { Reducción del Índice de } \\
\text { masa libre de grasa (IMLG) }\end{array}$ \\
\hline Opción 1 & $<18.5 \mathrm{~kg} / \mathrm{m} 2$ & $\mathrm{n} / \mathrm{a}$ & $\mathrm{n} / \mathrm{a}$ \\
\hline $\begin{array}{l}\text { Opción } 2 \\
\text { (combinada) }\end{array}$ & $<18.5 \mathrm{~kg} / \mathrm{m} 2$ & $\begin{array}{l}>10 \% \mathrm{o} \\
>5 \% \text { durante } \\
\text { meses }\end{array}$ & $\begin{array}{l}<15 \mathrm{~kg} / \mathrm{m} 2 \text { en mujeres } \\
<17 \mathrm{~kg} / \mathrm{m} 2 \text { en hombres }\end{array}$ \\
\hline
\end{tabular}

Tabla 1: DRE: Desnutrición Relacionada con la Enfermedad, n/a: no aplicable. La opción 2 combina: pérdida de peso e IMC reducido o IMLG. *La reducción del IMC es $<20$ o $<22 \mathrm{~kg} / \mathrm{m} 2$ en pacientes más jóvenes y mayores de 70 años, respectivamente.

Los cambios en la composición corporal con una pérdida de masa libre de grasa relacionada con la enfermedad en pacientes con sobrepeso no están necesariamente asociados con un IMC bajo, sin embargo, induce un "riesgo metabólico" que debe tenerse en cuenta para los pacientes que se someten a una cirugía mayor con especial consideración en los pacientes oncológicos. El exceso de macronutrientes de la obesidad puede coexistir con la malnutrición asociada a la inflamación crónica, la sarcopenia y la deficiencia de micronutrientes ${ }^{27,28}$. Los datos de grandes cohortes de pacientes postoperatorios sugieren que los obesos mórbidos (IM 4 C40 kg/m ${ }^{2}$ ) tienen más soporte ventilatorio postoperatorio, más infecciones y atención más prolongada en cuidados críticos ${ }^{29}$.

\section{El ayuno en el paciente quirúrgico.}

Factores como el riesgo potencial de aspiración de la vía aérea, dehiscencia anastomótica e íleo postoperatorio, fueron los impulsores del ayuno previo a la cirugía ${ }^{30}$. El objetivo de su prescripción era garantizar el vaciamiento gástrico y evitar la broncoaspiración en el momento de la inducción anestésica. La evidencia reciente y una comprensión más exacta de la motilidad gastrointestinal han cuestionado esta práctica. Desde hace varios años se conoce que el ayuno preoperatorio prolongado puede ser perjudicial, ya que induce estrés metabólico y afecta la función mitocondrial y la sensibilidad a la insulina ${ }^{31}$.Desde el 2006 el grupo europeo ERAS (EchancedRecovery After Surgery), enfatiza en la abreviación del ayuno ${ }^{32}$. La reducción de los períodos estándar de ayuno tiene poco o ningún efecto sobre el volumen o el pH del contenido gástrico $^{33,34}$. Un ayuno de 2 horas para líquidos claros es suficiente para asegurar un estómago vacío en la mayoría de los pacientes quirúrgicos elegidos adecuadamente, mientras que no debe consumirse ningún alimento sólido 6 horas antes de la inducción anestésica ${ }^{3536}$.

La resistencia insulínica (RI) iniciada en el ayuno preoperatorio prolongado aumenta con el trauma quirúrgico y es directamente proporcional a la magnitud de la cirugía. Luego de cirugías 
abdominales electivas y no complicadas, la RI puede durar hasta 3 semanas. Los resultados pueden optimizarse mediante un período de ayuno más corto precedido por la ingesta de carbohidratos ${ }^{37}$. Estudios han evidenciado que la sensibilidad postoperatoria a la insulina se preserva con bebidas ricas en carbohidratos (100 g la noche antes y 50 g 2 horas antes de la cirugía) ${ }^{38}$ o glucosa intravenosa (5 $\mathrm{mg} / \mathrm{kg} / \mathrm{min})^{39}$. Bilkuy otros. en una revisión sistemática de ensayos clínicos controlados aleatorizados, incluyendo a pacientes de cirugía abdominal, evidenciaron que las bebidas ricas en carbohidratos preoperatorias redujeron la RI y mejoraron una serie de marcadores subjetivos de bienestar del paciente, sin mayores tasas de eventos adversos ${ }^{40}$. En el mismo sentidoAwad y otros. en otro metaanálisis de 21 ensayos aleatorizados evidenciaron que la carga de carbohidratos por vía oral se asoció con menor duración de la estancia y menor RI en pacientes de cirugía abdominal mayor ${ }^{41}$. Si bien la carga preoperatoria de carbohidratos se ha incorporado en paquetes de cuidados para pacientes sometidos a cirugía colorrectal electiva, se requiere más evidencia antes de que esta práctica se establezca inequívocamente como beneficiosa y en otro tipo de pacientes ${ }^{42}$.

Las guías ESPEN 2017 recomiendan con un grado de recomendación alto, que los pacientes que no tienen riesgo específico de aspiración ingieran líquidos transparentes hasta dos horas antes de la anestesia y sólidos hasta seis horas antes del procedimiento. Consideran que el ayuno preoperatorio prolongado es innecesario en la mayoría de los pacientes ${ }^{43}$.

\section{Soporte nutricional durante el preoperatorio.}

La convalecencia óptima requiere suficiente reserva fisiológica preoperatoria para apoyar la movilización de las reservas de energía inducida por el estrés, apoyar la síntesis de proteínas de fase aguda y las células inmunes. El soporte nutricional especializado parece innecesario en pacientes normo nutridos o con riesgo nutricional bajo ${ }^{44,45}$; en pacientes médicos podría considerarse un sobretratamiento, dado queha demostrado poco efecto en los resultados clínicos en general ${ }^{46}$, excepto en las readmisiones no programadas según una revisión recientemente publicada ${ }^{47}$.

Los pacientes con alto riesgo nutricional o desnutrición severa se benefician claramente de la terapia nutricional preoperatoria. Schricker y otros demostraron una correlación significativa entre el grado de catabolismo encontrado antes de la cirugía y el efecto anabólico logrado con terapia nutricional perioperatoria $^{48}$. La evidencia sugiere que los suplementos de nutrición oral perioperatoria reducen las tasas de morbimortalidad, incluidas las infecciones de sitio quirúrgico ${ }^{49,50}$. En este sentido las guías internacionales recomiendan terapia nutricional para pacientes con desnutrición severa durante 7 a 14 días antes de una cirugía mayor electiva, aunque hay pruebas limitadas que respalden un período de tiempo específico. Las pautas de soporte de Nutrición Parenteral sugieren de 7 a 10 días, mientras que las pautas de soporte de Nutrición Enteral recomiendan de 10 a 14 días, incluso consideran diferir la cirugía. La Canadian Oncological Society afirma que, en el caso de cáncer de colon no urgente, es poco probable que las demoras en torno a 4 a 6 semanas desde el diagnóstico, con el objetivo de mejorar el estado nutricional, afecten negativamente la mortalidad quirúrgica o la supervivencia general ${ }^{51}$. Jie y otros. encontraron que los pacientes desnutridos experimentaron una reducción del $50 \%$ en la morbilidad postoperatoria con 5 a 7 días de terapia nutricional preoperatoria ${ }^{28}$. 


\section{Ruta nutricional.}

En cuanto a la ruta óptima de soporte nutricional preoperatorio, se prefiere la enteral por ser más fisiológica y menos costosa ${ }^{52}$. Las principales limitaciones para la prescripción de la nutrición parenteral preoperatoria incluyen las complicaciones del acceso venoso central, las infecciones relacionadas, la hiperglucemia, el síndrome de realimentación y los $\operatorname{costos}^{53,54}$. Un meta-análisis de 26 ensayos aleatorizados informó tasas reducidas de complicaciones cuando estando indicada, la nutrición parenteral se inició preoperatoriamente, particularmente en pacientes desnutridos, sin efecto sobre la mortalidad ${ }^{55}$. Un subgrupo limitado de pacientes con disfunción gastrointestinal puede beneficiarse de la nutrición parenteral preoperatoria durante 7 a 10 días $^{56}$.

\section{Inmunonutrición en cirugía.}

La cirugía es un insulto traumático que resulta en un período de inmunosupresión dentro de una población de pacientes que puede tener un sistema inmune debilitado relacionado con la desnutrición y otras comorbilidades ${ }^{57,58}$. La suplementación enteral o parenteral con nutrientes específicos como la arginina, la glutamina y los ácidos grasos omega-3 pueden modular la inflamación y regular positivamente las respuestas inmunitarias postoperatorias ${ }^{59,60}$. Al examinar pacientes sometidos a cirugías colorrectales sin deficiencias nutricionales, la Inmunonutrición dio lugar a mejores resultados ${ }^{61}$. El metaanálisis de Braga y otros. favoreció el uso de la Inmunonutrición en pacientes quirúrgicos electivos de alto riesgo. Sin embargo, muchos de los estudios incluidos se realizaron más de 2 décadas antes de su revisión y, por lo tanto, no reflejaron el valor de la Inmunonutrición en el contexto de los nuevos avances. En una revisión Burden y otros ${ }^{62}$ incluyeron siete ensayos que evaluaron la Inmunonutrición preoperatoria, de los cuales 6 fueron combinados en un meta-análisis. Estos estudios evidenciaron una reducción significativa de las complicaciones postoperatorias totales (RR 0,67; IC: 0,53 a 0,84). Se incluyeron tres ensayos que evaluaron la Nutrición Parenteral en un meta-análisis y se demostró una reducción significativa en las complicaciones postoperatorias (RR 0,64, IC del 95\%: 0,46 a 0,87) predominantemente en los pacientes desnutridos. Se incluyeron dos ensayos que evaluaron la nutrición enteral (RR 0.79, IC 95\% 0.56 a 1.10) y 3 ensayos que evaluaron los suplementos orales estándar (RR 1.01 IC 95\% 0.56 a 1.10), ninguno de los cuales mostró diferencias en los resultados primarios. Otros meta-análisis han encontrado una reducción en la duración de la estadía hospitalaria y la reducción de infecciones en pacientes que reciben suplementos parenterales de dipéptidos de glutamina ${ }^{63} \mathrm{o}$ arginina $^{64}$, cabe destacar que los estudios individuales incluidos en los meta-análisis tenían limitaciones metodológicas y estudiaban varios compuestos simultáneamente. Un meta-análisis realizado por Hegaziy otros. ${ }^{65}$ evaluó a 561 pacientes en ocho ensayos controlados aleatorios (ECA) de Inmunonutrición preoperatoria versus terapia enteral estándar, así como en nueve ECA de Inmunonutrición versus ningún suplemento. Se realizó un metaanálisis para los resultados informados, incluida la infección de la herida, las complicaciones infecciosas y no infecciosas, y la duración de la estadía. Durante su análisis, los autores no pudieron determinar un beneficio de la Inmunonutrición en comparación con el soporte nutricional enteral estándar.

Un interesante estudio de Moya y otros. ${ }^{66}$ aleatorizó a los pacientes a recibir nutrición oral estándar versus Inmunonutrición dentro de un protocolo ERAS. Ambos grupos fueron comparables en cuanto a edad, sexo, riesgo quirúrgico, comorbilidad y parámetros analíticos y nutricionales. Los autores 
informaron una disminución en el número total de complicaciones en el grupo de Inmunonutrición en comparación con el grupo de control, principalmente debido a una disminución significativa en las complicaciones infecciosas (23,8 frente a 10,7\%, P <0,0007). Recientemente Thornbladey otros. ${ }^{67}$ publicaron un estudio de cohorte prospectivo que incluyo a 3,375 pacientes que se sometieron a cirugía colorectal electiva, los datos de este estudio poblacional mostraron que la tasa de eventos adversos graves fue del $6.8 \%$ frente al $8.3 \%(\mathrm{p}=0.25)$ y la estadía prolongada fue del $13.8 \%$ frente al $17.3 \%(\mathrm{p}=0.04)$ en aquellos que recibieron y no recibieron Inmunonutrición preoperatoria, respectivamente. Aunque las diferencias en los eventos adversos graves no fueron significativas (RR 0.76; IC del 95\%: 0.49-1.16), la duración prolongada de la estadía $(\mathrm{RR}=0.77$; IC del 95\%: 0.58-1.01 $\mathrm{p}=0,05)$ fue menor en los que recibieron Inmunonutrición. Este estudio poblacional, con las limitaciones reconocidas por el autor, (se trató de una prueba no controlada y no se hizo un seguimiento del cumplimiento de las recomendaciones nutricionales ni se controló el volumen y los componentes consumidos), respalda los ensayos previos de Inmunonutrición, pero muestra una menor magnitud del beneficio.

Drover y colegas ${ }^{68}$ encontraron que los efectos beneficiosos de la nutrición perioperatoria eran superiores a la nutrición preoperatoria o postoperatoria sola. La mayoría de los otros metaanálisis y revisiones sistemáticas encuentran que Inmunonutrición preoperatoria y perioperatoria es superior a la que es administrada en el periodo postoperatorio solamente.

Probst P y otros. publicaron en 2017 un metaanálisis con más de 80 ECAs donde evidenciaron que laInmunonutrición redujo las complicaciones generales (OR 0.79; 95\% ci 0.66 a 0.94, P = 0,01), complicaciones infecciosas (OR 0,58 (0,51 a 0,66); P <0,001) y la estancia hospitalaria (diferencia de medias $-1,79(95 \%$ ci $-2,39$ a $-1,19)$ días, $\mathrm{P}<0,001)$ en comparación con los grupos de control. Sin embargo, estos efectos se redujeron luego de excluir los ensayos con un riesgo alto de sesgo.Los ensayos no financiados por la industria no informaron efectos positivos para las complicaciones generales (OR 1,13, 0,88 a 1,46, $\mathrm{P}=0,34)$, mientras que los financiados por la industria informaron efectos significativos (OR 0,66, 0, 48 a 0,91; $\mathrm{P}=0,01$ ).

A la luz de la evidencia actual, cuándo se administra, la Inmunonutrición debe iniciarse 5-7 días antes de la cirugía y continuar en el período postoperatorio ${ }^{69}$. En la tabla 5 se muestran los principales estudios de Inmunonutrición en el paciente quirúrgico.

Si bien el momento exacto, la cantidad y la composición de los inmunonutrientes aún no se han determinado, se requieren más estudios para seleccionar pacientes quirúrgicos para estas intervenciones nutricionales ${ }^{70}$.

Tabla 5 Resumen de los principales estudios de Inmunonutrición en el paciente quirúrgico.

\begin{tabular}{|c|c|c|c|c|c|}
\hline Autor & Año & Estudio & Pacientes & Metodología & Resultados \\
\hline $\begin{array}{c}\text { Braga M y } \\
\text { cols. }\end{array}$ & 2002 & ECA & $\begin{array}{c}\text { 200 pacientes } \\
\text { con neoplasma } \\
\text { colo-rectal } \\
\text { normo } \\
\text { nutridos. }\end{array}$ & $\begin{array}{c}\text { 4 grupos: (a) ingesta oral } \\
\text { durante 5 días antes de la } \\
\text { cirugía de una fórmula } \\
\text { enriquecida con arginina } \\
\text { y ácidos grasos n-3 }\end{array}$ & $\begin{array}{c}\text { La } \\
\text { administración } \\
\text { preoperatoria } \\
\text { reduce } \\
\text { infecciones. }\end{array}$ \\
\hline
\end{tabular}




\begin{tabular}{|c|c|c|c|c|c|}
\hline & & & & $\begin{array}{l}\text { (grupo preoperatorio); } \\
\text { (b) el mismo tratamiento } \\
\text { preoperatorio prolongado } \\
\text { después de la cirugía por } \\
\text { infusión yeyunal (grupo } \\
\text { peri-op); (c) ingesta oral } \\
\text { durante } 5 \text { días antes de la } \\
\text { cirugía de una fórmula } \\
\text { isoenergética estándar, } \\
\text { isonitrogenada (grupo } \\
\text { control); y (d) sin } \\
\text { suplementación antes y } \\
\text { después de la cirugía } \\
\text { (grupo convencional). }\end{array}$ & $\begin{array}{c}\text { La prolongación } \\
\text { postoperatoria } \\
\text { no tiene } \\
\text { beneficio } \\
\text { adicional. }\end{array}$ \\
\hline $\begin{array}{c}\text { Braga M y } \\
\text { cols. }^{71}\end{array}$ & 2002 & ECA & $\begin{array}{c}196 \text { pacientes } \\
\text { desnutridos } \\
\text { candidatos a } \\
\text { cirugía mayor } \\
\text { electiva por } \\
\text { cáncer del } \\
\text { tracto } \\
\text { gastrointestinal. }\end{array}$ & $\begin{array}{l}\text { Grupo control: recibió } \\
\text { NE postoperatoria (dieta } \\
\text { estándar dentro de las } 12 \\
\text { horas de la cirugía). } \\
\text { Grupo preoperatorio } \\
\text { recibió dieta líquida } \\
\text { enriquecida con arginina, } \\
\text { ácidos grasos omega-3 y } \\
\text { ARN por vo durante } 7 \\
\text { días, después de la } \\
\text { cirugía los pacientes } \\
\text { recibieron la misma } \\
\text { fórmula enteral estándar } \\
\text { que el grupo de control. } \\
\text { Un tercer grupo } \\
\text { (perioperatorio) recibió } \\
\text { durante } 7 \text { días la dieta } \\
\text { líquida enriquecida vo y } \\
\text { continuaron después de } \\
\text { la cirugía con la misma } \\
\text { fórmula enriquecida. }\end{array}$ & $\begin{array}{l}\text { La } \\
\text { Inmunonutrición } \\
\text { perioperatoria } \\
\text { reduce } \\
\text { complicaciones } \\
\text { y estancia } \\
\text { hospitalaria }\end{array}$ \\
\hline $\begin{array}{l}\text { Gianotti L } \\
\text { y cols. }{ }^{72}\end{array}$ & 2002 & ECA & $\begin{array}{l}305 \text { pacientes } \\
\text { desnutridos } \\
\text { candidatos a } \\
\text { cirugía por } \\
\text { cáncer del } \\
\text { tracto } \\
\text { gastrointestinal. }\end{array}$ & $\begin{array}{l}\text { (1) suplementación oral } \\
\text { durante } 5 \text { días antes de la } \\
\text { cirugía con una fórmula } \\
\text { enriquecida con arginina, } \\
\text { ácidos grasos omega-3 y } \\
\text { ARN, sin soporte } \\
\text { nutricional administrado }\end{array}$ & $\begin{array}{c}\text { La } \\
\text { suplementación } \\
\text { preoperatoria es } \\
\text { igual de efectiva } \\
\text { que la } \\
\text { perioperatoria. } \\
\text { Reducen }\end{array}$ \\
\hline
\end{tabular}




\begin{tabular}{|c|c|c|c|c|c|}
\hline & & & & $\begin{array}{c}\text { después de la cirugía } \\
\text { (grupo preoperatorio); } \\
\text { (2) el mismo tratamiento } \\
\text { preoperatorio más } \\
\text { infusión yeyunal } \\
\text { postoperatoria con la } \\
\text { misma fórmula } \\
\text { enriquecida (grupo } \\
\text { perioperatorio); y (3) sin } \\
\text { nutrición artificial antes } \\
\text { ni después de la cirugía } \\
\text { (grupo convencional). }\end{array}$ & $\begin{array}{c}\text { infecciones y } \\
\text { estadía } \\
\text { hospitalaria. }\end{array}$ \\
\hline $\begin{array}{l}\text { Senkal M y } \\
\text { cols. }^{73}\end{array}$ & 2002 & ECA & $\begin{array}{c}154 \text { pacientes } \\
\text { con neoplasias } \\
\text { malignas del } \\
\text { tracto } \\
\text { gastrointestinal } \\
\text { superior }\end{array}$ & $\begin{array}{c}5 \text { días de } \\
\text { Inmunonutrición oral } \\
\text { preoperatoria } \\
\text { (complementos con } \\
\text { arginina, ARN y ácidos } \\
\text { grasos omega 3) o una } \\
\text { dieta de control } \\
\text { isoenergética. NE } \\
\text { posoperatorio temprana } \\
\text { con Inmunonutrición o } \\
\text { una dieta control } \\
\text { isoenergética e } \\
\text { isonitrogenada por } \\
\text { yeyunostomía durante } 10 \\
\text { días. }\end{array}$ & $\begin{array}{l}\text { Disminuyó la } \\
\text { aparición de } \\
\text { infecciones } \\
\text { postoperatorias } \\
\text { y redujo los } \\
\text { costos }\end{array}$ \\
\hline $\begin{array}{l}\text { Zheng Y y } \\
\text { cols. }\end{array}$ & 2006 & $\begin{array}{c}\text { Revisión } \\
\text { Sistemática } \\
\text { y } \\
\text { metaanálisis }\end{array}$ & $\begin{array}{c}9 \text { ECAs con } \\
373 \text { pacientes }\end{array}$ & $\begin{array}{l}\text { Comparó NP estándar } \\
\text { con NP suplementada } \\
\text { con dipéptido de } \\
\text { glutamina en cirugía } \\
\text { abdominal. }\end{array}$ & $\begin{array}{l}\text { Disminuyó la } \\
\text { aparición de } \\
\text { infecciones y la } \\
\text { estancia } \\
\text { hospitalaria. }\end{array}$ \\
\hline $\begin{array}{l}\text { Cerantola } \\
\text { Y ycols }\end{array}$ & 2011 & $\begin{array}{c}\text { Revisión } \\
\text { Sistemática } \\
\text { y } \\
\text { metaanálisis }\end{array}$ & $\begin{array}{l}21 \text { ECAs, } 2730 \\
\text { pacientes se } \\
\text { incluyeron en } \\
\text { el metaanálisis. } \\
12 \text { ECAs } \\
\text { considerados } \\
\text { como estudios } \\
\text { de alta calidad. }\end{array}$ & $\begin{array}{l}\text { Inmunonutrición enteral } \\
\text { perioperatoria en cirugía } \\
\text { electiva gastrointestinal } \\
\text { mayor }\end{array}$ & $\begin{array}{c}\text { disminuye la } \\
\text { morbilidad y la } \\
\text { estancia } \\
\text { hospitalaria, } \\
\text { pero no la } \\
\text { mortalidad }\end{array}$ \\
\hline $\begin{array}{l}\text { Drover JW } \\
\text { y cols. }\end{array}$ & 2011 & $\begin{array}{c}\text { Revisión } \\
\text { sistemática }\end{array}$ & $\begin{array}{l}35 \text { ECAs con } \\
\text { pacientes de }\end{array}$ & $\begin{array}{l}\text { NE con Arginina (con o } \\
\text { sin otros agentes }\end{array}$ & $\begin{array}{l}\text { Reducción de } \\
\text { infecciones y }\end{array}$ \\
\hline
\end{tabular}




\begin{tabular}{|c|c|c|c|c|c|}
\hline & & $\begin{array}{c}\text { y } \\
\text { metaanálisis }\end{array}$ & $\begin{array}{c}\text { cirugía } \\
\text { electiva. }\end{array}$ & $\begin{array}{c}\text { inmunomoduladores) } \\
\text { versus NE estándar }\end{array}$ & $\begin{array}{c}\text { estadía } \\
\text { hospitalaria. }\end{array}$ \\
\hline $\begin{array}{c}\text { Burden S y } \\
\text { cols. }\end{array}$ & 2012 & $\begin{array}{c}\text { Revisión } \\
\text { sistemática } \\
\text { y } \\
\text { metaanálisis }\end{array}$ & $\begin{array}{c}\text { 7 ECAs con } \\
\text { pacientes de } \\
\text { cirugía } \\
\text { gastrointestinal, } \\
\text { 6 se } \\
\text { combinaron en } \\
\text { metaanálisis }\end{array}$ & $\begin{array}{c}\text { Inmunonutrición } \\
\text { parenteral, oral o enteral } \\
\text { perioperatoria. }\end{array}$ & $\begin{array}{c}\text { reducción de las } \\
\text { complicaciones } \\
\text { posoperatorias } \\
\text { totales con }\end{array}$ \\
L y cols. & 2017 & ECA & $\begin{array}{c}3375 \text { pacientes } \\
\text { de cirugía } \\
\text { electiva colo- } \\
\text { rectal. }\end{array}$ & $\begin{array}{c}\text { Inmunonutrición oral } \\
\text { (237 ml, 3 veces al día) } \\
\text { durante 5 días antes de la } \\
\text { cirugía. }\end{array}$ & $\begin{array}{c}\text { Reducción de } \\
\text { estadía y }\end{array}$ \\
complicaciones. \\
cols.
\end{tabular}

\section{Soporte nutricional postoperatorio.}

Los principios de la nutrición postoperatoria incluyen la alimentación enteral u oral temprana (incluida la proximal a una anastomosis) dentro de las primeras 24-48 horas ${ }^{74}$.

Luego de una cirugía abdominal no complicada, la función mioeléctrica del estómago regresa a patrones relativamente normales dentro de las 24-48 horas, el intestino delgado dentro de las 12-24 horas y el colon dentro de las 48-72 $\mathrm{h}^{75}$. La recuperación de la vía digestiva es clave en la recuperación del sistema inmunológico y, por lo tanto, fundamental para la recuperación tras la agresión.

Existe evidencia actual a favor de la nutrición enteral postoperatoria temprana, se ha demostrado que la reanudación de la nutrición oral o enteral dentro de las 24 horas posteriores a la cirugía es bien tolerada y segura, y no aumenta las tasas de dehiscencia anastomótica o íleo postoperatorio ${ }^{76,77,78}$. El metaanálisis de Mazaki y otros. ${ }^{79}$ que incluyó 29 estudios aleatorizados con 2552 pacientes, confirmaron los efectos beneficiosos de la nutrición enteral para una menor tasa de complicaciones infecciosas, fugas anastomóticas y una estancia hospitalaria más corta en pacientes de cirugía gastrointestinal. Los datos recientes de un ECA y un meta-análisis sugieren que la nutrición oral inmediata se puede administrar con seguridad en pacientes con anastomosis después de la gastrectomía parcial y total ${ }^{8081}$. En el mismo 
sentido, un meta-análisis de Osland y otros evaluó los resultados quirúrgicos después del soporte nutricional proporcionado proximal a la anastomosis dentro de las 24 horas de la cirugía gastrointestinal en comparación con el manejo postoperatorio tradicional. Quince estudios con un total de 1240 pacientes fueron analizados, los autores informaron una reducción estadísticamente significativa (45\%) en las probabilidades relativas de complicaciones postoperatorias totales en pacientes que recibieron alimentación posoperatoria temprana (OR 0.55, IC $0.35-0.87, \mathrm{p}=0,01$ ). No se observó ningún efecto de la alimentación temprana con relación a la dehiscencia anastomótica (OR 0.75; IC, 0.39-1.4, P = 0.39), mortalidad (OR 0.71; IC: 0.32-1.56, $\mathrm{P}=0.39$ ), número de días hasta expulsión de gases, ruidos intestinales o estancia reducida; sin embargo, la tendencia de los resultados clínicos favoreció la alimentación temprana. Los autores concluyen que la nutrición postoperatoria temprana se asocia con reducciones en las complicaciones totales y no afecta negativamente a los resultados ${ }^{82}$. Hasta el momento de esta revisión no encontramos datos controlados disponibles para pacientes con resección esofágica.

En este sentido, la ingesta oral, incluidos los líquidos claros, debe iniciarse dentro de las horas posteriores a la cirugía en la mayoría de los pacientes, según recomendaciones de las Guías ESPEN de $2017^{19}$.

En tanto que las directrices de la Sociedad Estadounidense de Nutrición Parenteral y Enteral (ASPEN) de 2016 recomiendan la nutrición enteral postoperatoria cuando sea factible dentro de las 24 horas ${ }^{83}$.

Se reserva la nutrición parenteral para pacientes de alto riesgo nutricional que no alcancen los requerimientos proteico-calóricos luego de optimizada la nutrición enteral, dentro de los 7-10 días posteriores a la cirugía. En casos seleccionados de disfunción gastrointestinal severa, isquemia intestinal, hemorragia digestiva, intestino corto o fistulas enterocutáneas de alto débito, la nutrición parenteral puede iniciarse precozmente ${ }^{1984}$.

\section{Pre-habilitación psico-física en el período preoperatorio.}

La población sometida a cirugía abdominal mayor es cada vez más añosa y frágil, con múltiples comorbilidades, muchos son obesos y físicamente no aptos. Estos desafíos para el paciente se ven agravados por problemas organizativos y de productividad, como el impulso para el alta postoperatoria temprana junto con la presión para reducir las tasas de readmisión ${ }^{85}$. Una disminución en la actividad física como resultado del envejecimiento o una enfermedad aguda resulta en un aumento significativo en el riesgo perioperatorio que puede atenuarse mediante intervenciones de ejercicio físico. Las intervenciones para mejorar la recuperación posquirúrgica generalmente se han dirigido a los períodos intraoperatorio y postoperatorio. Para los pacientes de alto riesgo a punto de someterse a una cirugía mayor, sin embargo, es probable que sea demasiado tarde ya que la mala aptitud física medida objetivamente está relacionada con malos resultados postoperatorios ${ }^{86}$.

Las mejoras recientes en los resultados postoperatorios se pueden atribuir a la centralización de la cirugía del cáncer, la introducción de técnicas quirúrgicas mínimamente invasivas y el desarrollo e implementación de programas de atención perioperatoria optimizados ${ }^{87}$. La Prehabilitación se basa en el principio de que el ejercicio estructurado en el período preoperatorio proporciona a los pacientes un "amortiguador fisiológico" para hacer frente al estrés de la cirugía; se asocia a reducción de las complicaciones postoperatorias y del deterioro funcional después de la cirugía ${ }^{88,89}$. Se ha demostrado que el entrenamiento de la fuerza aeróbica y muscular en pacientes quirúrgicos aumenta la resistencia, mejora 
marcadores objetivos de condición física y fuerza muscular. En este sentido una revisión sistemática demostró que un programa de ejercicios físicos preoperatorios es capaz de mejorar la capacidad funcional preoperatoria y reducir la tasa de complicaciones postoperatorias ${ }^{90}$. Más recientemente, dos revisiones sistemáticas han revisado la Prehabilitación del ejercicio en cirugía de cáncer abdominal, especialmente en cirugía colorrectal ${ }^{91,92}$. Morán y otros. ${ }^{93}$ revisaron los estudios que llevaban a cabo una Prehabilitación que consistía en entrenamiento de los músculos inspiratorios, ejercicios aeróbicos y ejercicios de resistencia. Estas modalidades de entrenamiento disminuyen la incidencia de complicaciones postoperatorias en pacientes sometidos a cirugía intraabdominal, este efecto fue más intenso cuando se comparó la Prehabilitación con la atención habitual o ejercicios de respiración solamente (OR 0.35; IC del 95\%: 0.17 a 0.71). Además, la Prehabilitación disminuyó significativamente la incidencia de complicaciones pulmonares postoperatorias (OR 0.27, IC 95\% 0.13-0.57), que se midieron como la principal complicación de interés en la mayoría de los estudios revisados.

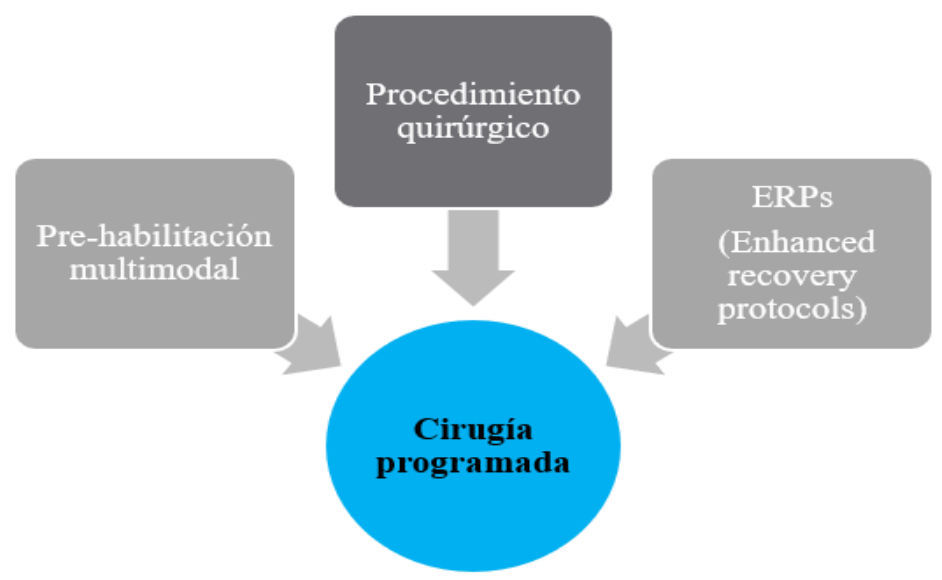

Figura 2. Enfoque multimodal perioperatorio.

La premisa del enfoque de Prehabilitación multimodal con un componente de nutrición, psicológico y ejercicio físico se basa en su sinergia. (Figura 2). Se ha sugerido que una combinación de asesoramiento nutricional individualizado, suplementos nutricionales y ejercicio es efectiva para mejorar la condición física y la calidad de vida en estudios de Prehabilitación ${ }^{94,95}$.Los futuros esfuerzos de investigación deberían explorar el potencial de combinar la Prehabilitación con los llamadosERPs(Enhancedrecoveryprotocols) para obtener mejoras adicionales en los resultados postoperatorios $^{96}$. La Prehabilitación es el precursor lógico de un ERP y aborda la brecha en los aspectos preoperatorios del ERP que actualmente se limitan a la orientación preoperatoria,nutrición y manejo de la anemia.

\section{Conclusiones}

Los pacientes quirúrgicos en riesgo nutricional tienen una morbimortalidad, tiempo de hospitalización, tasas de reingresos y costos asociados con sus episodios de internación significativamente más altos. Estos pacientes deben ser identificados tempranamente, pero el método sigue siendo incierto, 
en ellos se recomienda iniciar la terapia nutricional (preferiblemente por vía enteral) sin demora. Los períodos de ayuno prolongado deben minimizarse y la nutrición debe comenzar tan pronto como sea posible después de la cirugía. La terapia nutricional debe iniciarse y ajustarse de acuerdo con cada paciente en particular, no existe el concepto de talla única. La ruta óptima, la cantidad y el momento del soporte en el período perioperatorio sigue siendo foco de la investigación. La Inmunonutrición reduce las complicaciones generales, las complicaciones infecciosas y acorta la estancia hospitalaria, sin efecto sobre la supervivencia. La mejora del estado nutricional y la recuperación funcional, incluida la calidad de vida son los objetivos nutricionales más importantes en el postoperatorio alejado; por lo tanto, la identificación de intervenciones para optimizar la capacidad funcional preoperatoria antes de la cirugía mayor debería ser una prioridad. Se debe enfatizar el cambio a evaluaciones e intervenciones nutricionales preventivas en lugar de reactivas. Se ha demostrado que las intervenciones nutricionales perioperatorias adecuadas mejoran los resultados en cirugía.

\section{Bibliografía}

\footnotetext{
${ }^{1}$ Ben-Ishay O, Gertsenzon H, Mashiach T, Kluger Y, Chermesh I. Malnutrition in surgical wards: a plea for concern. Gastroenterol. Res. Pract. 2011; 2011: doi: 10.1155/2011/840512.
}

${ }^{2}$ Ho JW, Wu AH, Lee MW, Lau SY, Lam PS, Lau WS, et al. Malnutrition risk predicts surgical outcomes in patients undergoing gastrointestinal operations: results of a prospective study. Clin. Nutr. 2014; 34: 679-84.

${ }^{3}$ Planas Vila M, Álvarez Hernández J, García de Lorenzo A, Celaya Pérez S, León Sanz M, García-Lorda $\mathrm{P}$, et al. The burden of hospital malnutrition in Spain: Methods and development of the PREDyCES1 Study. Nutr Hosp. 2010; 25:1020-4.

${ }^{4}$ National Alliance for Infusion Therapy and the American Society for Parenteral and Enteral Nutrition Public Policy Committee and Board of Directors. Disease-related malnutrition and enteral nutrition theraphy: A significant problem with a cost-effective solution. Nutr Clin Pract. 2010; 25:548-54.

${ }^{5}$ Waitzberg DL, Caiaffa WT, Correia MI. Hospital malnutrition: The Brazilian national survey (IBRANUTRI): a study of 4000 patients. Nutrition 2001; 17(7-8): 573-80.

${ }^{6}$ Correia MI, Perman MI, Waitzberg DL. Hospital malnutrition in Latin America: A systematic review. Clin Nutr 2017;36:958-67.

${ }^{7}$ Braunschweig C, Gomez S, Sheean PM: Impact of declines in nutritional status on outcomes in adult patients hospitalized for more than 7 days. J Am Diet Assoc 2000; 100:1316-22

${ }^{8}$ Desborough JP. The stress response to trauma and surgery. Br. J. Anaesth. 2000; 85:109-17.

${ }^{9}$ Gustafsson Ulf O, Ljungqvist O. Perioperative nutritional management in digestive tract surgery. Curr Opin Clin Nutr Metab Care. 2011;14:504-9. 
${ }^{10}$ van Stijn MF, Korkic-Halilovic I, Bakker MS, van der Ploeg T, van Leeuwen PA, Houdijk AP. Preoperative nutrition status and postoperative outcome in elderly general surgery patients: a systematic review. J Parenter Enteral Nutr 2013;37:37-43.

${ }^{11}$ Aahlin EK, Tranø G, Johns N, Horn A, Søreide JA, Fearon KC, et al. Risk factors, complications and survival after upper abdominal surgery: a prospective cohort study. BMC Surg 2015; 15:83

12 Sorensen J, Kondrup J, Prokopowicz J, Schiesser M, Krahenbuhl L, Meier R, et al. EuroOOPS: an international, multicentre study to implement nutritional risk screening and evaluate clinical outcome. Clin Nutr 2008;27: 340-9

${ }^{13}$ Kassin MT, Owen RM, Perez SD, et al. Risk factors for 30- day hospital readmission among general surgery patients. Journal of the American College of Surgeons 2012; 215:322-30.

${ }^{14}$ Wilmore DW. Metabolic response to severe surgical illness: overview. World J Surg 2000; 24:705-11

${ }^{15}$ Scholl R, Bekker A, Babu R. Neuroendocrine and immune responses to surgery . Internet J Anesthesiol 2012;30:3. Disponible en: http://ispub.com/IJA/30/3/14145

${ }^{16}$ Carli F, Zavorsky GS. Optimizing functional exercise capacity in the elderly surgical population. Curr Opin Clin Nutr Metab Care. 2005;8(1):23-32.

${ }^{17}$ Strickland A, Brogan A, Krauss J, Martindale R, Cresci G. Is the use of specialized nutritional formulations a cost-effective strategy? A national database evaluation. Journal J Parenter Enteral Nutr 2005; 29(Suppl. 1): S81- 91. Doi 10.1177/01486071050290S1S81

${ }^{18}$ Melchior JC, Préaud E, Carles J, Brami M , Duru G, Fontaine E, et al. Clinical and economic impact of malnutrition per se on the postoperative course of colorectal cancer patients. Clin Nutr 2012; 31: 896902.

${ }^{19}$ Weimann A, Braga M, Carli F, Higashiguchi T, Hübner M, Klek S, et al. ESPEN guideline: Clinical nutrition in surgery. Clin Nutr, 2017; 36):623-50.

${ }^{20}$ Kondrup J, Allison SP, Elia M, Vellas B, Plauth M, Educational and Clinical Practice Committee, European Society of Parenteral and Enteral Nutrition (ESPEN). ESPEN guidelines for nutrition screening 2002. Clin Nutr 2003;22: 415e21.

${ }^{21}$ Hennessey DB, Burke JP, Ni-Dhonochu T, Shields C, Winter DC, Mealy K. Preoperative hypoalbuminemia is an independent risk factor for the development of surgical site infection following gastrointestinal surgery: a multi-institutional study. Ann. Surg. 2010;252:325-9.

${ }^{22}$ Kudsk KA, Tolley EA, DeWitt RC, Janu PG, Blackwell AP, Yeary S, King BK. Preoperative albumin and surgical site identify surgical risk for major postoperative complications. J Parenter Enteral Nutr 2003;27:1-9. 
${ }^{23}$ Mueller C, Compher C, Ellen DM. A.S.P.E.N. clinical guidelines: nutrition screening, assessment, and intervention in adults. J Parenter Enteral Nutr 2011; 35: 16-24.

${ }^{24}$ Kondrup J, Rasmussen HH, Hamberg O, Stanga Z. Nutritional risk screening (NRS 2002): a new method based on an analysis of controlled clinical trials. Clin Nutr 2003; 22: 321-36.

${ }^{25}$ Gupta D, Vashi PG, Lammersfeld CA, Braun DP. Role of nutritional status in predicting the length of stay in cancer: a systematic review of the epidemiological literature. Ann Nutr Metab 2011;59:96-106.

${ }^{26}$ Cederholm T, Bosaeus I, Barazzoni R, Bauer J, Van Gossum A, Klek S, et al. Diagnostic criteria for malnutrition - an ESPEN consensus statement. Clin Nutr 2015; 34:335-40.

${ }^{27}$ Evans DC, Martindale RG, Kiraly LN, Jones CM. Nutrition optimization prior to surgery. Nutr Clin Pract 2014; 29:10-21.

${ }^{28}$ Toh SY, Zarshenas N, Jorgensen J. Prevalence of nutrient deficiencies in bariatric patients. Nutrition 2009; 25:1150- 6 .

${ }^{29}$ Valentijn TM, Galal W, Tjeertes EK, Hoeks SE, Verhagen HJ, Stolker RJ. The obesity paradox in the surgical population. Surgeon 2013; 11:169-76.

${ }^{30}$ Maltby JR. Fasting from midnight - the history behind the dogma. Best Pract Res ClinAnaesthesiol 2006; 20: 363-78.

${ }^{31}$ Awad S, Lobo DN. What's new in perioperative nutritional support? Curr Opin Anaesthesiol 2011;24:339-48

${ }^{32}$ Wind J1, Polle SW, Fung Kon Jin PH, Dejong CH, von Meyenfeldt MF, Ubbink DT et al. Systematic review of enhanced recovery programmes in colonic surgery. Br J Surg. 2006;93(7):800-9.

${ }^{33}$ Soreide E, Eriksson LI, Hirlekar G, et al. Pre-operative fasting guidelines: an update. Acta Anaesthesiol Scand 2005; 49:1041-7.

${ }^{34}$ Brady M, Kinn S, Stuart P. Preoperative fasting for adults to prevent perioperative complications. The Cochrane Database of Systematic Reviews 2003; 4: CD004423.

35 American Society of Anesthesiologists. Practice guidelines for preoperative fasting and the use of pharmacologic agents to reduce the risk of pulmonary aspiration: application to healthy patients undergoing elective procedures: an updated report by the American Society of Anesthesiologists

Committee on Standards and Practice Parameters. Anesthesiol 2011;114:495-511.

\footnotetext{
${ }^{36}$ Lambert E, Carey S. C. Practice Guideline Recommendations on Perioperative Fasting: a systematic review. J
} Parenter Enteral Nutr 2015. doi 10.1177/0148607114567713. 
${ }^{37}$ Nygren J, Thorell A, Ljungqvist O. Are there any benefits from minimizing fasting and optimization of nutrition and fluid management for patients undergoing day surgery? Curr Op Anaesthesiol. 2007;20: $540-4$.

${ }^{38}$ Nygren J, Soop M, Thorell A, Efendic S, Nair KS, Ljungqvist O. Preoperative oral carbohydrate administration reduces postoperative insulin resistance. Clin Nutr. 1998;17:65-71.

${ }^{39}$ Ljungqvist O, Thorell A, Gutniak M, Haggmark T, Efendic S. Glucose infusion instead of preoperative fasting reduces postoperative insulin resistance. J Am Coll Surg 1994;178:329-36.

${ }^{40}$ Bilku DK, Dennison AR, Hall TC, Metcalfe MS, Garcea G. Role of preoperative carbohydrate loading: a systematic review. Ann R Coll Surg Engl 2014;96:15-22.

${ }^{41}$ Awad S, Varadhan KK, Ljungqvist O, Lobo DN. A meta-analysis of randomised controlled trials on preoperative oral carbohydrate treatment in elective surgery. Clin Nutr 2013;32:34-44.

42 Gustafsson UO, Scott MJ, Schwenk W, et al. Guidelines for perioperative care in elective colonic surgery: Enhanced Recovery After Surgery (ERAS) Society recommendations. World J Surg 2013;37: 259-84.

${ }^{43}$ Weimann A, Braga M, Carli F, Higashiguchi T, Hübner M, Klek S, et al. ESPEN guideline: Clinical nutrition in surgery. Clin Nutr 2017;36:623-50.

${ }^{44}$ Huhmann MB, August DA. Nutrition support in surgical oncology. Nutr Clin Pract 2009; 24: 520-6.

${ }^{45}$ Burden S, Todd C, Hill J, Lal S. Pre-operative nutrition support in patients undergoing gastrointestinal surgery. The Cochrane Database of Systematic Reviews 2012; 11: CD008879

${ }^{46}$ Arias S, Bruzzone I, Blanco V, Inchausti M, García F, Casavieja G, et al. Reconocimiento y soporte nutricional precoz en pacientes hospitalizados desnutridos. Nutr Hosp. 2008;23(4):348-53

${ }^{47}$ Bally MR, Blaser Yildirim PZ, Bounoure L, et al. Nutritional support and outcomes in malnourished medical inpatients: a systematic review and meta-analysis. JAMA Intern Med. 2016;176(1):43-53.

${ }^{48}$ Schricker T, Wykes L, Meterissian S, Hatzakorzian R, Eberhart L, Carvalho G, et al. The anabolic effect of perioperative nutrition depends on the patient's catabolic state before surgery. Ann Surg 2013; 257:155-9

${ }^{49}$ Stratton RJ, Elia M: Who benefits from nutritional support: What is the evidence? Eur J Gastroenterol Hepatol 2007; 19:353-8

${ }^{50}$ Stratton RJ, Elia M: A review of reviews: A new look at the evidence for oral nutritional supplements in clinical practice. Clin Nutr Suppl 2007; 2:5-23

${ }^{51}$ Simunovic M, Rempel E, Thériault ME, Baxter NN, Virnig BA, Meropol NJ, et al: Influence of delays to nonemergent colon cancer surgery on operative mortality, disease-specific survival and overall survival. Can J Surg 2009; 52:E79-86 
${ }^{52}$ McClave SA, Kozar R, Martindale RG, et al. Summary points and consensus recommendations from the North American Surgical Nutrition Summit. J Parenter Enteral Nutr 2013; 37(Suppl. 5): 99S-105S.

53 Evans DC, Martindale RG, Kiraly LN, Jones CM. Nutrition optimization prior to surgery. Nutr Clin Pract 2014;29:10-21.

${ }^{54}$ Abunnaja S, Cuviello A, Sanchez JA. Enteral and parenteral nutrition in the perioperative period: state of the art. Nutrients 2013;5:608-23.

${ }^{55}$ Heyland DK, Montalvo M, MacDonald S, Keefe L, Su XY, Drover JW. Total parenteral nutrition in the surgical patient: a meta-analysis. Can J Surg 2001;44:102- 11.

${ }^{56}$ Ali Abdelhamid Y, Chapman M, and Deane A. Peri-operative nutrition. Anaesthesia 2016, 71 (Suppl. 1), 9-18

${ }^{57}$ Braga M. The 2015 ESPEN ArvidWretlind lecture. Evolving concepts on perioperative metabolism and support, Clin Nutr 2015. Doi 0.1016/j.clnu.2015.12.012

${ }^{58}$ Leandro-Merhi VA, de Aquino JL. Determinants of malnutrition and post-operative complications in hospitalized surgical patients. J Health Popul Nutr 2014;32(3):400-10.

59 Braga M, Wischmeyer PE, Drover J, Heyland DK. Clinical evidence for pharmaconutrition in major elective surgery. J Parenter Enteral Nutr 2013; 37(Suppl. 5): 66S-72S.

${ }^{60}$ Braga M. Perioperative immunonutrition and gut function. Curr Opin Clin Nutr Metab Care 2012; 15: 485-8.

${ }^{61}$ Braga M, Gianotti L, Vignali A, et al. Preoperative oral arginine and n-3 fatty acid supplementation improves the immunometabolic host response and outcome after colorectal resection for cancer. Surgery 2002;132(5):805-14.

${ }^{62}$ Burden S, Todd C, Hill J, Lal S. Pre-operative nutrition support in patients undergoing gastrointestinal surgery. The Cochrane Database of Systematic Reviews 2012; 11: CD008879.

63 Zheng YM, Li F, Zhang MM, Wu XT. Glutamine dipeptide for parenteral nutrition in abdominal surgery: a meta-analysis of randomized controlled trials. World J Gastroenterol 2006; 12: 7537-41.

${ }^{64}$ Drover JW, Dhaliwal R, Weitzel L, Wischmeyer PE, Ochoa JB, Heyland DK. Perioperative use of arginine-supplemented diets: a systematic review of the evidence. J Am Coll Surg 2011; 212: 385-99.

65 Hegazi RA, Hustead DS, Evans DC. Preoperative standard oral nutrition supplements vs immunonutrition: results of a systematic review and meta-analysis. J Am Coll Surg. 2014; 219(5):107887.

${ }^{66}$ Moya P, Soriano-Irigaray L, Ramirez JM, Garcea A, Blasco O, Blanco FJ, et al. Perioperative Standard Oral Nutrition Supplements Versus Immunonutrition in Patients Undergoing Colorectal Resection in an 
Enhanced Recovery (ERAS) Protocol: A Multicenter Randomized Clinical Trial (SONVI Study). Medicine (Baltimore). 2016 May;95(21): e3704. doi: 10.1097/MD.0000000000003704.

${ }^{67}$ Thornblade LW, Varghese TK Jr, Shi X, Johnson EK, Bastawrous A, Billingham RP, et al. Preoperative Immunonutrition and Elective Colorectal Resection Outcomes. Dis Colon Rectum. 2017;60(1):68-75.

${ }^{68}$ Cerantola Y, Hübner M, Grass F, Demartines N, Schäfer M. Immunonutrition in gastrointestinal surgery. Br J Surg 2011;98(1):37-48.

${ }^{69}$ McClave SA, Kozar R, Martindale RG, et al. Summary points and consensus recommendations from the North American Surgical Nutrition Summit. J Parenter Enteral Nutr 2013;37(Suppl. 5):99S-105S.

${ }^{70}$ Gupta R, Senagore A. Immunonutrition within enhanced recovery after surgery (ERAS): an unresolved matter. Perioper Med (Lond). 2017; 11;6:24. doi: 10.1186/s13741-017-0080-5.

${ }^{71}$ Braga M, Gianotti L, Nespoli L, Radaelli G, Di Carlo V. Nutritional approach in malnourished surgical patients: a prospective randomized study. Arch Surg. 2002;137(2):174-80.

${ }^{72}$ Gianotti L, Braga M, Nespoli L, et al. A randomized controlled trial of preoperative oral supplementation with a specialized diet in patients with gastrointestinal cancer. Gastroenterology. 2002; 122(7):1763e1770.

${ }^{73}$ Senkal M, Zumtobel V, Bauer KH, et al. Outcome and cost-effectiveness of perioperative enteral immunonutrition in patients undergoing elective upper gastrointestinal tract surgery: a prospective randomized study. Arch Surg Chic Ill 1960). 1999;134(12):1309e1316.

${ }^{74}$ Lambert E, Carey S. Practice guideline recommendations on perioperative fasting: a systematic review. J Parenter Enteral Nutr 2015; doi 10.1177/0148607114567713.

75 Martindale RG, McClave SA, Taylor B, Lawson CM. Perioperative nutrition: what is the current landscape? J Parenter Enteral Nutr 2013; 37(5 Suppl.): 5S-20S.

${ }^{76}$ Lewis SJ, Andersen HK, Thomas S. Early enteral nutrition within $24 \mathrm{~h}$ of intestinal surgery versus later commencement of feeding: a systematic review and meta-analysis. J Gastrointest Surg 2009; 13:569e75.

${ }^{77}$ Steed HL, Capstick V, Flood C, Schepansky A, Schulz J, Mayes DC. A randomized controlled trial of early versus "traditional” " postoperative oral intake after major abdominal gynecologic surgery. Am J of Obstet Gynecol 2002;186: 61-5.

${ }^{78}$ Andersen HK, Lewis SJ, Thomas S. Early enteral nutrition within 24 h of colorectal surgery versus later commencement of feeding for postoperative complications. The Cochrane Database of Systematic Reviews 2006; 4: CD004080.

${ }^{79}$ Mazaki T, Ebisawa K. Enteral versus parenteral nutrition after gastrointestinal surgery: a systematic review and meta-analysis of randomized controlled trials in the English literature. J Gastrointest Surg 2008;12: 739 - 55 . 
${ }^{80}$ Hur H, Kim SG, Shim JH, Song KY, Kim W, Park CH, et al. Effect of early oral feeding after gastric cancer surgery: a result of randomized clinical trial. Surgery 2011; 149:561e8.

${ }^{81}$ Willcutts KF, Chung MC, Erenberg CL, Finn KL, Schirmer BD, Byham-Gray LD. Early oral feeding as compared with traditional timing of oral feeding after upper gastrointestinal surgery. Ann Surg 2016; 264:54e63.

${ }^{82}$ Osland E, Yunus RM, Khan S, Memon MA. Early versus traditional postoperative feeding in patients undergoing resectional gastrointestinal surgery: a meta-analysis. JPEN J Parenter Enteral Nutr. 2011; 35(4):473-87. doi: 10.1177/0148607110385698.

${ }^{83}$ McClave SA, Taylor BE, Martindale RG, Warren MM, Johnson DR, Braunschweig C, et alA.S.P.E.N. Board of Directors, American College of Critical Care Medicine, Society of Critical Care Medicine. Guidelines for the provision and assessment of nutrition support therapy in the adult critically ill patient: society of critical care medicine (SCCM) and American society for parenteral and enteral nutrition (A.S.P.E.N.). J Parenter Enteral Nutr 2016;40: 159e211.

${ }^{84}$ McClave SA, Martindale R, Taylor B, Gramlich L. Appropriate use of parenteral nutrition through the perioperative period. Journal of Parenteral and Enteral Nutrition 2013; 37(Suppl. 5):73S-82S.

85 Pearse RM, Harrison DA, James P, Watson D, Hinds C, Rhodes A et al. Identification and characterisation of the high-risk surgical population in the United Kingdom. Crit Care 2006; 10: R81.

${ }^{86}$ Moran J, Wilson F, Guinan E, McCormick P, Hussey J, Moriarty J. Role of cardiopulmonary exercise testing as a risk-assessment method in patients undergoing intra-abdominal surgery: a systematic review. Br J Anaesth. 2016;116(2):177-91.

${ }^{87}$ Adamina M, Kehlet H, Tomlinson GA, Senagore AJ, Delaney CP. Enhanced recovery pathways optimize health outcomes and resource utilization: a meta-analysis of randomized controlled trials in colorectal surgery. Surgery 2011; 149: 830-40.

${ }^{88}$ Carli F, Charlebois P, Stein B, Feldman L, Zavorsky G, Kim DJ et al. Randomized clinical trial of prehabilitation in colorectal surgery. Br J Surg 2010; 97: 1187-97.

${ }^{89}$ Adamsen L, Quist M, Andersen C, Moller T, Herrstedt J, Kronborg D, et al. Effect of a multimodal high intensity exercise intervention in cancer patients undergoing chemotherapy: randomized controlled trial. BMJ 2009; 339: b3410.

90 Santa Mina D, Clarke H, Ritvo P, Leung YW, Matthew AG, Katz J, et al. Effect of total-body prehabilitation on postoperative out-comes: a systematic review and meta-analysis. Physiotherapy 2014;100(3):196-207.

${ }^{91}$ Bruns ERJ, van den Heuvel B, Buskens CJ, van Duijvendijk P, Festen S, Wassenaar EB, et al. The effects of physical prehabilitation in elderly patients undergoing colorectal surgery: a systematic review. Color Dis. 2016;18(8):O267-77. 
${ }^{92}$ Hijazi Y, Gondal U, Aziz O. A systematic review of prehabilitation programs in abdominal cancer surgery. Int J Surg. 2017;39:156-62.

${ }^{93}$ Moran J, Guinan E, McCormick P, Larkin J, Mockler D, Hussey J, et al. The ability of prehabilitation to influence postoperative outcome after intra-abdominal operation: a systematic review and metaanalysis. Surgery. 2016;160:1189-201.

${ }^{94}$ Li C, Carli F, Lee L, Charlebois P, Stein B, Liberman AS, et al. Impact of a trimodal prehabilitation program on functional recovery after colorectal cancer surgery: a pilot study. Surg Endosc. 2013;27(4):1072-82.

${ }^{95}$ Gillis C, Loiselle S-E, Fiore JF, Awasthi R, Wykes L, Liberman AS, et al. Prehabilitation with whey protein supplementation on perioperative functional exercise capacity in patients undergoing colorectal resection for cancer: a pilot double-blinded randomized placebocontrolled trial. J AcadNutr Diet. 2015;116:802-12.

${ }^{96}$ Moorthy, K. and Wynter-Blyth, V. Prehabilitation in perioperative care. Br J Surg. 2017;104: 802-3. doi:10.1002/bjs.10516 